\title{
Where are the limits of the centrosome?
}

\author{
Irina B. Alieva ${ }^{\mathrm{a}}$ and Rustem E. Uzbekov ${ }^{\mathrm{b}, \mathrm{c}}$ \\ ${ }^{a}$ A. N. Belozersky Institute of Physico-Chemical Biology, Moscow State University, \\ Moscow, Russia; \\ ${ }^{\mathrm{b}}$ Laboratory of Cell Biology and Electron Microscopy, Faculty of Medicine, François \\ Rabelais University, Tours, France; \\ ${ }^{c}$ Faculty of Bioengineering and Bioinformatics, Moscow State University, Moscow, \\ Russia
}

\begin{abstract}
The centrosome is a key component of the cell is involved in the processes of cell division, cell motility, intracellular transport, organization of the microtubules (MT) network and the production of cilia and flagella. The peculiarity of this organelle is that its boundaries are not clearly defined, the centrioles at the center of the centrosome are surrounded by electron dense pericentriolar material, the size and protein composition of this centrosome component experiences significant transformation during the cell cycle. It has been shown in this study that within the centrosome different proteins occupy different areas corresponding to: MT nucleation region (defined as gammatubulin-stained area), regulatory region (defined as kinase pEg2-stained area) and motor proteins region (kinesin-like motor XlEg5-stained area). The boundary of $\mathrm{pEg} 2$ is near 1.3 times greater while XlEg5 is 3.0 times greater than that of gamma-tubulin. Thus, the size of the centrosome, determined according to the structural electron microscopy (EM) analysis (about $1 \mu \mathrm{m}$ ) corresponds to the regulatory proteins area, but the actual functional centrosome size defined at the motor proteins region, is more than twice the size.
\end{abstract}

KEYWORDS. centriole, centrosome, microtubules, mitosis, pericentriolar material, prophase

\section{INTRODUCTION}

The centrosome plays a crucial role in a number of cellular processes during the interphase, and is also involved in the organization and orientation of the mitotic spindle during cell division. It's location at the geometric center of interphase cells, the concentration of

Correspondence to: Rustem E. Uzbekov; Email: rustem.uzbekov@univ-tours.fr; Irina B. Alieva; Email: irina_alieva@belozersky.msu.ru

Received February 22, 2016; Revised March 15, 2016; Accepted March 16, 2016.

Color versions of one or more of the figures in this article can be found online at www.tandfonline.com/kbia. 
various regulatory proteins in the centrosome area and centrosome-organized radial microtubule (MT) array (which is the scaffold for motor proteins involved in intracellular transport) suggests that this unique cellular structure may function as the regulation and distribution center, that manages the dynamic morphology of the animal cell.

The centrosome, a cellular organelle visible at optical level as a dense granule, has a very complicated ultrastructure, described in detail in numerous electron microscopic studies. ${ }^{1}$ The centrosome is made up of a pair of centrioles (cylindrical structures, consisting of 9 microtubule triplets), which are surrounded by pericentriolar material. The average length of a mature centriolar cylinder (in vertebrates) is 0.3 $0.5 \mu \mathrm{m}$, the diameter is about $0.2 \mu \mathrm{m}$.

The structural integrity of the centrioles is not really based on the stability of MT triplets, as on the durability of their surrounding materialcentriolar matrix. During the transition to mitosis the centrosome is reorganized, its expansion is significantly greater than the limits of the matrix of the centrioles to form a pericentriolar halo.

Considering the above, we may draw a conclusion that the boundaries of the centrosome are not defined by the size of its most prominent morphological elements (centrioles and associated structures). Apparently, the boundaries of the centrosome (depending on the cell cycle stage) coincide with the boundaries of the amorphous material surrounding the structural components of the centrosome and containing centrosomal proteins.

The centrosomal proteins can be classified by several parameters, such as: their localization in the centrosome, the time spent centrosome, their functions. Another classification uses the structural-temporal organization of proteins based on different protein affinity with the basic components of the centrosome. ${ }^{2}$

According to the proposed classification, centrosome boundaries can be defined by the size of structural domains occupied by various centrosomal proteins. In this study we measured the parameters of these domains by immunofluorescence and electron microscopy (EM). Based on these measurements, a model of centrosome organization was proposed, where different functional activities (nucleation, regulation and motor activity) occupy distinct but overlapping concentric zones around the centrioles.

\section{MATERIALS AND METHODS}

\section{Cell culture}

XL2 (Xenopus laevis) cells were cultured at $25^{\circ} \mathrm{C}$, without $\mathrm{CO}_{2}$ in L-15 Leibovitz medium (Life Technologies, Inc.) supplemented with $10 \%$ fetal calf serum (Bio Times) and antibiotic-antimycotic (Life Technologies, Inc.) (penicillin (100 Units/ ml), streptomycin $(100 \mathrm{mg} / \mathrm{ml})$, and amphotericin $(25 \mathrm{mg} / \mathrm{ml}))$ in $25 \mathrm{~cm} 2$ cell flasks (Falcon) or 12-well cell culture plates (Corning Inc.) on coverslips.

\section{Immunofluorescence staining}

Xenopus XL2 cells were grown on glass coverslips and fixed through immersion in cold $\left(-20^{\circ} \mathrm{C}\right) 100 \%$ methanol for $6 \mathrm{~min}$, washed 3 times in a phosphate-buffered saline solution (PBS) (pH 7,2), and blocked in PBS containing $3 \%$ bovine serum albumin (BSA) for $30 \mathrm{~min}$. MT were detected with mouse anti- $\beta$-tubulin monoclonal antibody (clone TUB2.1, Sigma), pEg2 was detected with the mouse monoclonal antibodies $1 \mathrm{Cl},{ }^{3}$ gamma-tubulin was detected with a rabbit polyclonal antibody, ${ }^{4} \mathrm{XIEg} 5$ was detected with rabbit purified polyclonal antibody. ${ }^{5}$ The antibodies were revealed by incubation with Texas red-conjugated goat anti-mouse $\mathrm{IgG}$ (Interchim) and fluorescein isothiocyanate (FITC)-conjugated goat anti-rabbit IgG (Interchim). All antibody reagents were diluted in PBS containing 1\% BSA and the incubations were performed at room temperature for 60 min. Cells were rinsed in PBS containing $1 \%$ BSA between each incubation and mounted in Mowiol containing antifade.

For immunofluorescence microscopy, as well as for electron microscopy, prophase cells were selected, using phase contrast microscopy, in asynchronous cell culture. For electron microscopy prophase cells were previously found under the light microscope in prepared 
Epon samples, than the pyramids containing prophase cells on their surface were manufactured for the serial sectioning.

\section{Immunofluorescence microscopy}

Zeiss Axiolab microscope (AXIOVERT 35) equipped with phase contrast and epifluorescence was used for indirect immunofluorescence microscopy. Samples were observed using x100/1.25 NA Achroplan objective and special double filter set for the observation of fluorescence at 488 and $594 \mathrm{~nm}$ wavelengths simultaneously. Cells were photographed using a Nikon 601 camera using high resolution films. The films were scanned at $4800 \mathrm{dpi}$ and analyzed using Adobe Photoshop software. Stage micrometer was photographed using x100/1.25 NA Achroplan objective for accurate calibration of the resulting images and for obtaining the scale bar for the images.

\section{Electron microscopy}

The cells were rinsed in PBS and fixed in cold $\left(-20^{\circ} \mathrm{C}\right) 100 \%$ methanol, washed 3 times in PBS and post-fixed with $1 \% \mathrm{OsO}_{4}$. Then cell were stained with uranyl acetate, dehydrated and embedded in an Epon 812 (Sigma). Serial ultra-thin $(70 \mathrm{~nm})$ sections were obtained with the LKB-V ultramicrotome and mounted on single slot grids. The sections were examined using a Philips electron microscope operating at $80 \mathrm{kV}$ and photographed.

\section{Data analysis}

Quantitative analysis was obtained by measuring profiles of fluorescence intensity of proteins of our interest using ImageJ (NIH) software, linked to an Excel (Microsoft Corp., Redmond, WA) spreadsheet. Data obtained were analyzed statistically using ImageJ and Excel software. Statistical analysis was done with unpaired Student's t tests, and data are expressed as Mean \pm SEM as indicated in figure legends. $\mathrm{P}$ values $\leq 0.05$ were considered to be significant.

\section{RESULTS AND DISCUSSION}

For a comparative study we chose the prophase of mitosis, 2 divergent early mitotic centrosomes at this stage had a regular spherical structure. Thus the measured sizes are not dependent on the centrosome's orientation, which is relative to the visual angle. The most important function of the centrosome is MT nucleation. Minus ends of MT are fixed on the centrosome and their plus ends are directed toward the cell periphery. MT growth is initiated by gamma-tubulin-based multiprotein complexes. The MT nucleating function of gamma-tubulin has motivated us to use this protein as a marker for the central region of the centrosome in our experiments. The centrosome organizes intracellular transport by using 2 types of motors that are capable to move molecules and whole organelles along the MT in centrifugal (kinesins) and centripetal (dyneins) directions. In our experiments we used antibodies against the kinesin-like protein XlEg5 to localize the area of motor proteins.

The centrosome as a cellular organelle without membrane boundaries concentrates all centrosomal components including its regulatory molecules to provide high efficiency of their interactions. These interactions may in part be performed by centripetal transport along the centrosome-attached MTs. In the present study $\mathrm{pEg} 2$ was used as a marker of regulatory centrosomal molecules. This protein kinase has fundamental importance during the interphaseto-mitosis transition. ${ }^{3} \mathrm{pEg} 2$ is a member of Aurora-A family which interacts with numerous regulatory centrosomal proteins. ${ }^{2}$ Thus, from the localization of $\mathrm{pEg} 2$ within the centrosome we implicitly define the localization of function-related regulatory proteins.

We compared the diameter of the zones occupied by selected protein-markers in the prophase centrosome with the data of EM analysis and stained them with antibodies against $\beta$-tubulin, which revealed that MT were associated with the centrosome. The results are shown in Figure 1.

With double labeling of gamma-tubulin / $\mathrm{pEg} 2$ showed that in contrast to interphase ${ }^{3}$ in prophase the centrosome $\mathrm{pEg} 2$ occupies a 
larger area than gamma-tubulin (Fig. 1A-D). This region was in the center of prophase MT aster (Fig. 1I). In all cases we observed one spot only, and did not see spatially distinct zones corresponding to mother and daughter centrioles. Electron microscopic study also showed that both centrioles were surrounded by pericentriolar material (Fig. 1J). The diameter of the gamma-tubulin area was $0,67 \pm 0,11$ (n $=24) \mu \mathrm{m}$. The diameter of $\mathrm{pEg} 2$ area was $0,88 \pm 0,18(\mathrm{n}=21) \mu \mathrm{m}$. Thus, according to our data, the "nucleation region" was near 1.3 times smaller than the "regulatory region."

It was shown that Aurora-A interacts and colocalizes with proteins: Ajuba, ${ }^{6}$ TPX $2,{ }^{7}$ centrosomin, ${ }^{8}$ D-TACC ${ }^{9}$ and HEF1. ${ }^{10}$ Hence, we can assume that the area in which Aurora-A kinase is revealed completely or partially

FIGURE 1. Localization of different proteins in prophase centrosome area in XL2 cells. (A-C) pEg2/gamma-tubulin double immunofluorescent labeling; (D) the profiles of the fluorescence intensity of $\mathrm{pEg} 2$ and gamma-tubulin immunofluorescence (vertical axis, arbitrary units) as a function of the distance from the geometric center of the fluorescent area (horizontal axis, $\mu \mathrm{m}) ;(\mathrm{E}-\mathrm{G}) \mathrm{pEg} 2 /$ XIEg5 double immunofluorescent labeling; $(H)$ the profile of the fluorescence intensity of $\mathrm{pEg} 2$ and XIEg5 immunofluorescence (vertical axis, arbitrary units) as a function of distance from the geometric center of the fluorescent area (horizontal axis, $\mu \mathrm{m}$ ); (I) double $\beta$-tubulin/gamma-tubulin immunofluorescent labeling; $(\mathrm{J})$ ultrastructure of centrosome region in prophase XL2 cell, TEM analysis; $(K)$ representative scheme of different centrosomal proteins localization. Horizontal dashed line $(D, H)$ shows the fluorescence level for which the measurement of the fluorescent spot radius was made. Scale bar $1 \mu \mathrm{m}$.
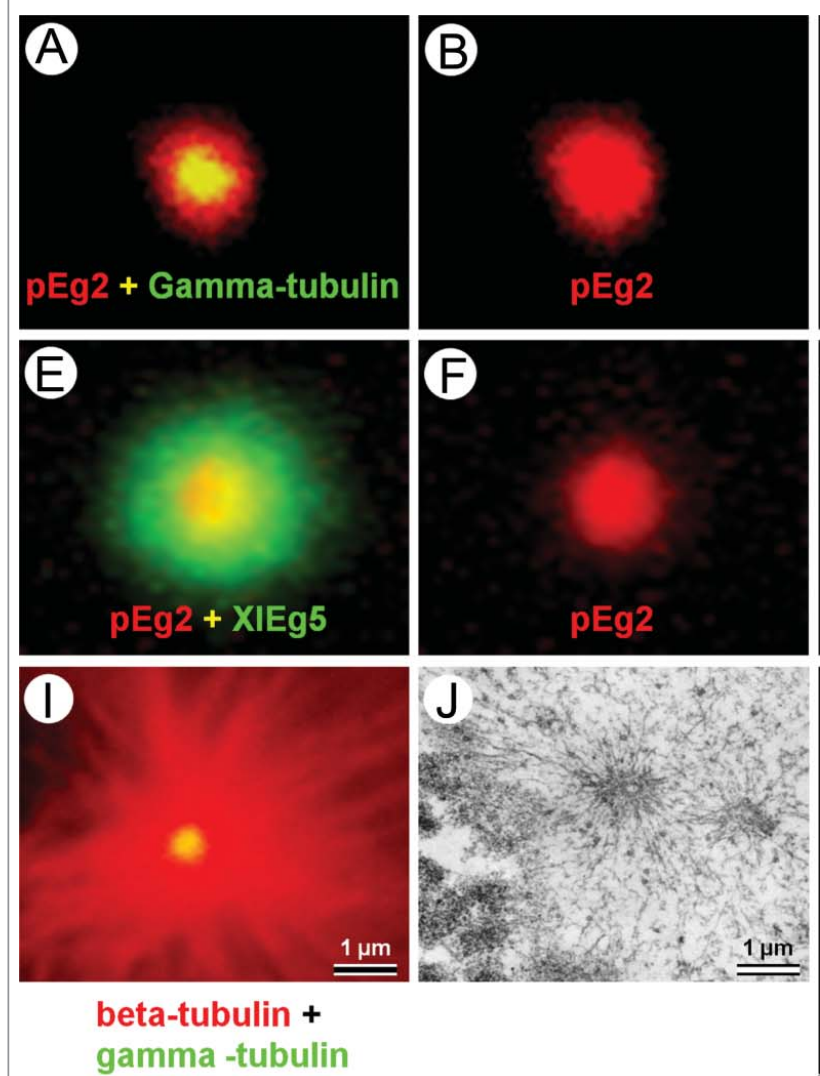

beta-tubulin +
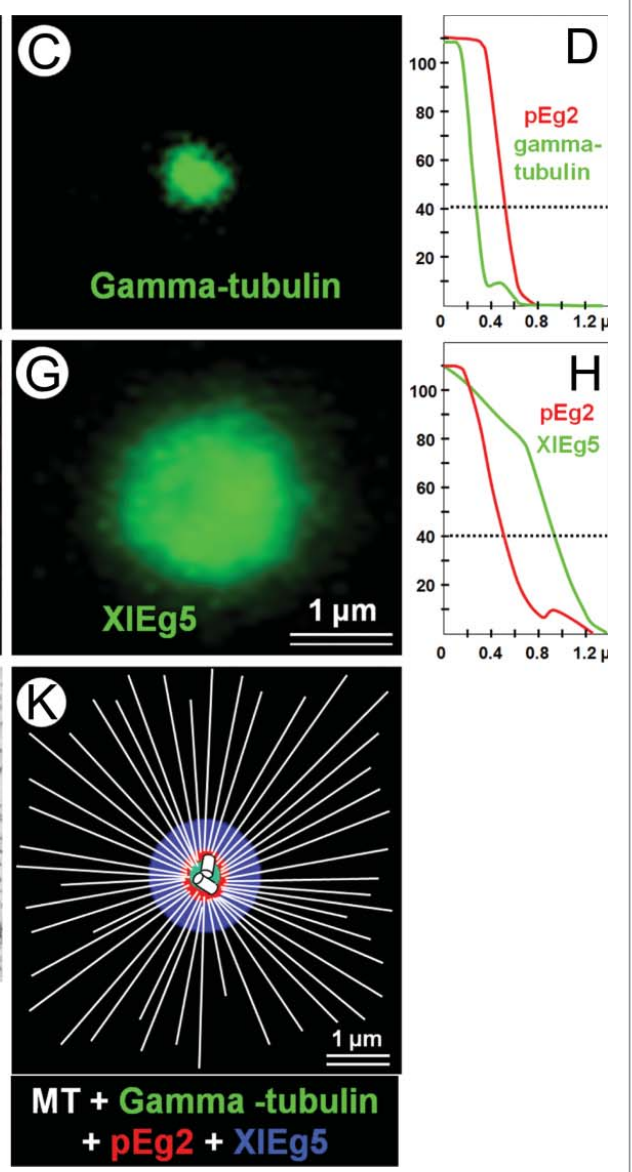
coincides with the areas occupied by its partners in the volume of the centrosome.

Kinesin-like protein XlEg5 first appears between separating centrosomes in $\mathrm{G}_{2}$-phase of the cell cycle. ${ }^{11}$ In prophase cells it is localized around each of 2 centrosomes. XlEg5 is a plus-end MT-related motor and its activity is associated with the separation of the 2 centrosomesfuture poles of mitotic spindle. The suggested mechanism presupposes that a MT emanating from one centrosome interacts with an antiparallel MT from the other centrosome. Dimers or tetramers of XlEg5 interact simultaneously with oppositely directed MTs and push them apart. We can assume that the area of localization of this protein must be sufficiently large to increase the probability of interaction with MT.

Indeed, the diameter of XlEg5 "motors region" was $1.99 \pm 0.15 \mu \mathrm{m} \quad(\mathrm{n}=10)$ (Fig. 1E-H), 2.97 times bigger than zone of gamma-tubulin and 2.26 times bigger than zone of $\mathrm{pEg} 2$. The MT nucleation zone is located in the very center of the centrosome. It is surrounded by the regulatory zone which is spatially coincident with the electron dense mitotic halo detectable at an ultrastructural level. The widest area is the motor area that covers an even larger volume (Fig. 1G, H).

Since mitotic halo is absent in the interphase cells, centrosomal proteins are more compactly located. The structural illumination microscopy method allowed the estimation of the size of the interphase centrosome in Drosophila cells. ${ }^{12}$ The authors reported that before clouds of PCM appear, its proteins are closely associated with interphase centrioles in 2 tube-like layers: an inner layer (diameter $380 \pm 70 \mathrm{~nm}$ ) occupied by centriolar microtubules, Sas-4, Spd-2, Polo kinase; and an outer layer (diameter $540 \pm 30 \mathrm{~nm}$ ) including Pericentrin-like protein, Asterless and Plk4 kinase. Centrosomin and gamma-tubulin associate with this outer tube in $\mathrm{G}_{2}$ cells. The results of this study cannot be compared with our data directly-in addition to the differences caused by the stages of the cell cycle selected for the analysis, the centrosome organization in insects is different from those in vertebrates. For example, for the mammalian centrosomes approximately the same quantity of gamma-tubulin is found in mother and daughter centrioles in $\mathrm{G}_{1}$-phase, and the presence of a certain quantity of gamma-tubulin is present in procentrioles. ${ }^{4}$

Considering the limitations of epifluorescence microscopy in this paper we evaluated different areas within the centrosome (nucleation region, regulatory region and motor proteins region) relative to one another, but the development of new imaging technique (STORM, STED, SIM) will allow to perform the measurement of actual centrosome size more accurately.

\section{CONCLUSION}

Returning to the question that we asked in the headline of this article - where are the limits of the centrosome - we can conclude that the actual functional boundaries of the centrosome defined as the region of localization of motor proteins. This region is about twice as large as the size of the mitotic halo which is visible with the electron microscope and corresponds to the area of centrosome associated motor protein distribution.

\section{DISCLOSURE OF POTENTIAL CONFLICTS OF INTEREST}

No potential conflicts of interest were disclosed.

\section{ACKNOWLEDGMENTS}

We thank Dr. Igor Kireev (Moscow State University, Moscow) and Dr. Claude Prigent (Institut de Génétique et Développement de Rennes) for critical comments on the manuscript. We thank Ann Rose Cook for English proofreading of the text.

\section{FUNDING}

This work was supported by Russian Foundation for Basic Research (Grants \#12-0400488 and \#15-04-08550) to I.B.A. 


\section{REFERENCES}

[1] Wheatley DN. The centriole: a central enigma of cell biology. Amsterdam/New York: Elsevier Biomedical Press; 1982.

[2] Alieva IB, Uzbekov RE. The centrosome is a polyfunctional multiprotein cell complex. Biochemistry (Moscow) 2008; 73: 626-3; PMID:18620528; http:// www.protein.bio.msu.ru/biokhimiya/contents/v79/ full/79091188.html

[3] Roghi C, Giet R, Uzbekov R, Morin N, Chartrain I, Le Guellec R, Couturier A, Doree M, Philippe M, Prigent $\mathrm{C}$. The Xenopus protein kinase $\mathrm{pEg} 2$ associates with the centrosome in a cell cycle-dependent manner, binds to the spindle microtubules and is involved in bipolar mitotic spindle assembly. J Cell Sci 1998 ; 111:557-2; PMID:9454730; http://jcs.biol ogists.org/content/joces/111/5/557.full.pdf

[4] Komarova YA, Ryabov EV, Alieva IB, Uzbekov RE, Uzbekova SV, Vorobjev IA. Polyclonal antibodies against human gamma-tubulin stain centrioles in mammalian cells from different tissues. Membr Cell Biol 1997; 10:503-3; PMID:9225254.

[5] Giet R, Uzbekov R, Cubizolles F, Le Guellec K, Prigent $\mathrm{C}$. The Xenopus laevis aurora-related protein kinase pEg2 associates with and phosphorylates the kinesin-related protein XlEg5. J Biol Chem 1999; 274: 15005-3; PMID:10329703; http://www.jbc. org/content/274/21/15005.full.pdf + html

[6] Hirota T, Kunitoku N, Sasayama T, Marumoto T, Zhang D, Nitta M, Hatakeyam, K, Saya H. Aurora$\mathrm{A}$ and an interacting activator, the LIM protein
Ajuba, are required for mitotic commitmnt in human cells. Cell 2003; 114:585-8; PMID: 13678582.

[7] Eyers PA, Erikson E, Chen LG, Maller JL. A novel mechanism for activation of the protein kinase Aurora A. Curr Biol 2003; 13: 691-7; PMID:18620528; http://dx.doi.org/10.1016/S0960-9822(03)00166-0

[8] Terada Y, Uetake Y, Kuriyama R. Interaction of Aurora-A and centrosomin at the microtubulenucleating site in Drosophila and mammalian cells. J Cell Biol 2003; 162:757-763; PMID:12939255; http://www.jcb.org/cgi/doi/10.1083/jcb.200305048

[9] Giet R, McLean D, Descamps S, Lee MJ, Raff JW, Prigent C, Glover DM. Drosophila Aurora A kinase is required to localize D-TACC to centrosomes and to regulate astral microtubules. J Cell Biol 2002; 156: 437-1; PMID:11827981; PMCID: PMC2173350; http://www.jcb.org/cgi/doi/10.1083/jcb.200108135

[10] Pugacheva EN, Jablonski SA, Hartman TR, Hensk EP, Golemis EA. HEF1-dependent Aurora A activation induces disassembly of the primary cilium. Cell 2007; 129:1351-3; PMID:17604723; http://dx.doi. org/10.1016/j.cell.2007.04.035

[11] Giet R, Descamps S, Lee MJ, Raff JW, Prigent C, Glover DM. Drosophila Aurora A kinase is required to localize D-TACC to centrosomes and to regulate astral microtubules. J Cell Biol 2002; 156:437-1; PMID:11827981; http://www.ncbi.nlm.nih.gov/ pmc/articles/PMC2173350/pdf/0108135.pdf

[12] Fu J, Glover DM. Structured illumination of the interface between centriole and peri-centriolar material. Open Biol 2012; 28: 120104; PMID:22977736; http://dx.doi.org/10.1098/rsob.120104 